\title{
Is Invasive Mucinous Breast Carcinoma A Rare Variant With Neuroendocrine Differentiation?
}

\author{
Ayesha Asghar, Aresha Masood Shah
}

\begin{abstract}
We want to bring your attention towards the rare variant of breast cancer hence promoting its early detection and screening. The main advantage of early diagnosis is reduced tumor size without distant dissemination at the initial treatment.
\end{abstract}

Neuroendocrine malignancies are heterogenous with neuronal and endocrinal properties. Neuroendocrine cells are present throughout the body and hence neuroendocrine tumors (NETS) can arise from any anatomic region. Although breast cancer is the most frequently diagnosed cancer in women, breast NETS are very rare. With a prevalence of $1-2$ cases per 10,000 women, breast NETS make up only $0.1 \%$ of all breast cancers and less than $1 \%$ of all NETS ${ }^{1}$. In other histopathological subtypes of breast carcinomas, invasive ductal carcinoma is the most common breast tumor reported worldwide of which mucinous (colloid) variant is the rare histological subtype, accounts for $1-7 \%$ of all invasive carcinomas $^{2}$. However, $30 \%$ of invasive breast tumor are seen with nneuroendocrine differentiation, and is most commonly associated with mucinous and solid papillary carcinomas and therefore treated similar to another invasive carcinoma of breast ${ }^{3}$.

Mucinous carcinoma is well circumcised and have soft gelatinous consistency. It has two subtypes, pure mucinous or mixed type. Pure mucinous carcinoma is a rare cancer which most commonly occur in older women and less than $1 \%$ occurs in younger than 35 years of age ${ }^{4}$. Pure type is composed of entirely mucinous carcinoma with two further subtypes, hypocellular and hypercellular variants. Axillary lymph node involvement is commonly rare. In a study of Kashiwagi, demographical data of 71 patients with mucinous carcinoma of the breast have suggests tumor size ranged from $0.5 \mathrm{~cm}-12.3 \mathrm{~cm}$ with mean tumor diameter- $3.1 \mathrm{~cm}^{6}$. Mucinous carcinoma with neuroendocrine differentiation shows good prognosis but tumors which are expressed of HER 2 neu shows negative prognosis and has increased risk of recurrence and metastasis. Primary neuroendocrine tumors of breast have worst prognosis due to lack of long-term survival data hence it's difficult to make a prognosis for this carcinoma ${ }^{5}$.

Diagnosis of mucinous carcinoma depends upon tumor or lump detection on self-examination or through mammogram

Ayesha Asghar
Final Year Student MBBS
Jinnah Sindh Medical University, Karachi
Email: ayeshaasghar98@gmail.com
Aresha Masood Shah
Final Year Student MBBS
innah Sindh Medical University, Karachi
Received: 09-Mar-2020
Accepted: 16-Apr-2020

or MRI apart from that Ultrasound guided percutaneous core biopsy should also be ordered. Other signs include painful retracted nipples with discharge that isn't breast milk, unusual changes in the appearance of breast and milk.

Treatment plan include mastectomy or lumpectomy which do not differ from primary protocol treatment of all breast carcinoma. Adjuvant therapy such as chemotherapy, extra cranial stereotactic radiation therapy followed by endocrine hormonal therapy such as tamoxifen or an aromatase inhibitor which block the effect of estrogen. As mucinous carcinomas are estrogen and progesterone receptor positive hence it is likely to be effective ${ }^{2}$. Apart from this most mucinous are also negative for HER 2 neu so they should not be treated with Herceptin. Neuroendocrine tumors of the breast have a significantly higher rate of local and distant recurrence and lower overall survival than Invasive ductal carcinoma ${ }^{2}$. However, in the NCCN guidelines Mucinous Breast Carcinoma is regarded as the "a histological type with a favorable prognosis"

Mucinous carcinoma of breast being the rarest form of invasive carcinoma which is mostly associated with neuroendocrine differentiation should be kept into consideration while diagnosing the cancers of breast in old age women particularly postmenopausal women. Management plan for invasive carcinoma with Neuroendocrine differentiation is a challenge particularly the diagnosis owing to the variably present and cytological features of $\mathrm{NTE}^{4}$. Due to its rarity, data on molecular characteristics is limited hence more research should be done on this carcinoma to enhance the diagnostic and treatment modalities for upcoming doctors.

\section{REFERENCES:}

1. Saeed A, Rehman A, Zaidi SAH, Shaukat T, Jamil K, Abdullah K. Neuroendocrine carcinoma of breast. J Coll Physicians Surg Pak. 2011;21(6):371-3.

2. Al Haddad H AA, Abdel Hadi M. . Mucinous breast carcinoma: Report of four cases and review of the literature. Clinical and Diagnostic Pathology. 2017;1(4).

3. Rosen L GP. Neuroendocrine Tumors of the Breast. Archives of Pathology \& Laboratory Medicine. 2017;141(11):157781.

4. Yang M, Li X, Pang CH, Huang LP. Pure Mucinous Breast Carcinoma: A Favorable Subtype. Breast Care. 2013;8(1):569.

5. Varadharajan E, Priya S, Prakash G, Mugundan A, Easwaramurthi P. Mucinous Carcinoma of the Breast with Neuroendocrine Differentiation. Iranian journal of pathology. 2015;10(3):231-6.

6. Kashiwagi et al.: Clinical significance of the subclassification of 71 cases mucinous breast carcinoma. SpringerPlus 2013 2:481doi:10.1186/2193-1801-2-481. 